\title{
Herschel/HIFI observations of spectrally resolved methylidyne signatures toward the high-mass star-forming core NGC $63341^{\star, \star \star}$
}

\author{
M. H. D. van der Wiel ${ }^{1,2}$, F. F. S. van der Tak ${ }^{2,1}$, D. C. Lis $^{3}$, T. Bell ${ }^{3}$, E. A. Bergin ${ }^{4}$, C. Comito ${ }^{5}$, M. Emprechtinger ${ }^{3}$, \\ P. Schilke ${ }^{5,6}$, E. Caux ${ }^{7,8}$, C. Ceccarelli $9,10,11$, A. Baudry ${ }^{10,11}$, P. F. Goldsmith ${ }^{12}$, E. Herbst ${ }^{13}$, W. Langer ${ }^{12}$, S. Lord ${ }^{15}$, \\ D. Neufeld ${ }^{16}$, J. Pearson ${ }^{12}$, T. Phillips ${ }^{3}$, R. Rolffs ${ }^{6,5}$, H. Yorke ${ }^{12}$, A. Bacmann ${ }^{9,10,11}$, M. Benedettini ${ }^{14}$, G. A. Blake ${ }^{3}$, \\ A. Boogert ${ }^{15}$, S. Bottinelli 7,8 , S. Cabrit ${ }^{17}$, P. Caselli ${ }^{18}$, A. Castets ${ }^{9,10,11}$, J. Cernicharo ${ }^{19}$, C. Codella ${ }^{20}$, A. Coutens ${ }^{7,8}$, \\ N. Crimier ${ }^{9,19}$, K. Demyk ${ }^{7,8}$, C. Dominik ${ }^{21,22}$, P. Encrenaz ${ }^{17}$, E. Falgarone ${ }^{17}$, A. Fuente ${ }^{23}$, M. Gerin ${ }^{17}$, F. Helmich ${ }^{2}$, \\ P. Hennebelle ${ }^{17}$, T. Henning ${ }^{24}$, P. Hily-Blant ${ }^{9}$, T. Jacq ${ }^{10,11}$, C. Kahane ${ }^{9}$, M. Kama ${ }^{21}$, A. Klotz ${ }^{7,8}$, B. Lefloch ${ }^{9}$,
} A. Lorenzani ${ }^{20}$, S. Maret ${ }^{9}$, G. Melnick ${ }^{25}$, B. Nisini2 ${ }^{26}$, S. Pacheco ${ }^{9}$, L. Pagani ${ }^{17}$, B. Parise ${ }^{5}$, M. Salez ${ }^{17}$, P. Saraceno ${ }^{14}$, K. Schuster ${ }^{27}$, A. G. G. M. Tielens ${ }^{28}$, C. Vastel ${ }^{7,8}$, S. Viti ${ }^{29}$, V. Wakelam ${ }^{10,11}$, A. Walters ${ }^{7,8}$, F. Wyrowski ${ }^{5}$, K. Edwards ${ }^{30}$, J. Zmuidzinas ${ }^{3}$, P. Morris ${ }^{3}$, L. A. Samoska ${ }^{12}$, and D. Teyssier ${ }^{31}$

(Affiliations are available on page 5 of the online edition)

Received 31 May 2010 / Accepted 7 July 2010

\section{ABSTRACT}

Context. In contrast to the more extensively studied dense star-forming cores, little is known about diffuse gas surrounding star-forming regions. Aims. We study the molecular gas in the Galactic high-mass star-forming region NGC 6334I, which contains diffuse, quiescent components that are inconspicuous in widely used molecular tracers such as $\mathrm{CO}$.

Methods. We present Herschel/HIFI observations of methylidyne (CH) toward NGC 6334I observed as part of the "Chemical HErschel Survey of Star forming regions" (CHESS) key program. HIFI resolves each of the six hyperfine components of the lowest rotational transition $\left(J=\frac{3}{2}-\frac{1}{2}\right)$ of $\mathrm{CH}$, observed in both emission and absorption.

Results. The $\mathrm{CH}$ emission features appear close to the systemic velocity of NGC6334I, while its measured $F W H M$ linewidth of $3 \mathrm{~km} \mathrm{~s}^{-1}$ is smaller than previously observed in dense gas tracers such as $\mathrm{NH}_{3}$ and $\mathrm{SiO}$. The $\mathrm{CH}$ abundance in the hot core is $\sim 7 \times 10^{-11}$, two to three orders of magnitude lower than in diffuse clouds. While other studies find distinct outflows in, e.g., $\mathrm{CO}$ and $\mathrm{H}_{2} \mathrm{O}$ toward NGC 6334I, we do not detect any outflow signatures in $\mathrm{CH}$. At least two redshifted components of cold absorbing material must be present at -3.0 and $+6.5 \mathrm{~km} \mathrm{~s}^{-1}$ to explain the absorption signatures. We derive a $\mathrm{CH}$ column density $\left(N_{\mathrm{CH}}\right)$ of $7 \times 10^{13}$ and $3 \times 10^{13} \mathrm{~cm}^{-2}$ for these two absorbing clouds. We find evidence of two additional absorbing clouds at +8.0 and $0.0 \mathrm{~km} \mathrm{~s}^{-1}$, both with $N_{\mathrm{CH}} \approx 2 \times 10^{13} \mathrm{~cm}^{-2}$. Turbulent linewidths for the four absorption components vary between 1.5 and $5.0 \mathrm{~km} \mathrm{~s}^{-1}$ in $F W H M$. We constrain the physical properties and locations of the clouds by matching our CH absorbers with the absorption signatures seen in other molecular tracers.

Conclusions. In the hot core, molecules such as $\mathrm{H}_{2} \mathrm{O}$ and $\mathrm{CO}$ trace gas that is heated and dynamically influenced by outflow activity, whereas the $\mathrm{CH}$ molecule traces more quiescent material. The four $\mathrm{CH}$ absorbing clouds have column densities and turbulent properties that are consistent with those of diffuse clouds: two are located in the direct surroundings of NGC 6334, and two are unrelated foreground clouds. Local density and dynamical effects influence the chemical composition of the physical components of NGC 6334, which causes some components to be seen in $\mathrm{CH}$ but not in other tracers, and vice versa.

Key words. stars: formation - ISM: molecules - ISM: individual objects: NGC 6334I

\section{Introduction}

Most studies of star-forming regions focus on dense gas in the cores, while much less is known about surrounding diffuse clouds. Methylidyne $(\mathrm{CH})$ can be used as a tracer of low density clouds, since its column density is known to correlate with the total molecular column density in diffuse clouds (Van Dishoeck \& Black 1986; Liszt \& Lucas 2002; Sheffer et al. 2008; Chastain et al. 2010). In contrast, most carbon is locked in CO in higher density environments $\left(n\left(\mathrm{H}_{2}\right) \gtrsim 10^{4} \mathrm{~cm}^{-3}\right)$, resulting in a lower fractional abundance of $\mathrm{CH}$ (e.g., Polehampton et al. 2005).

\footnotetext{
* Herschel is an ESA space observatory with science instruments provided by European-led Principal Investigator consortia and with important participation from NASA.

$\star \star$ Acknowledgements (page 5) are only available in electronic form at http://www . aanda.org
}

Interstellar $\mathrm{CH}$ was first observed in its electronic transitions in the visual (Adams 1941), and later the $\Lambda$-doublet at $3.3 \mathrm{GHz}$ (Rydbeck et al. 1973; Turner \& Zuckerman 1974). The accurate determination of a $\mathrm{CH}$ abundance from its radio lines is complicated by population inversion effects, while its pure rotational transitions lie in the far-infrared and are unobservable from the ground. While the rotational spectrum of $\mathrm{CH}$ has been studied since the 1970s (see Davidson et al. 2001), the first astronomical detection of a rotational transition of $\mathrm{CH}$ (at $149 \mu \mathrm{m}$ ) was reported by Stacey et al. (1987) in Sgr B2. With the Heterodyne Instrument for the Far-Infrared (HIFI, de Graauw et al. 2010) onboard the Herschel Space Observatory (Pilbratt et al. 2010), it is now possible to observe the ground-state rotational transition of $\mathrm{CH}$ at 533 and $537 \mathrm{GHz}$, free from atmospheric interference and with unprecedented spectral resolution. 
In this Letter, we study spectrally resolved signatures of the $\mathrm{CH}^{2} \Pi_{3 / 2} J=3 / 2-1 / 2$ transition, with an upper level energy of $26 \mathrm{~K}$, toward NCG 6334I, which exhibits emission from the hot core itself, and absorption due to cold foreground clouds. NGC 6334I is a high-mass star-forming core at a distance of $1.7 \mathrm{kpc}$ belonging to the luminous NGC 6334 complex of molecular clouds. The "I" source is composed of several dense subcores (Hunter et al. 2006) with a total mass of $\sim 200 M_{\odot}$ and a bolometric luminosity of several $10^{5} L_{\odot}$ (Sandell 2000). It is rich in molecular lines (e.g., Schilke et al. 2006). Several foreground clouds have been identified along the line of sight toward NGC 6334 by Brooks \& Whiteoak (2001) and Beuther et al. (2005). We use Herschel/HIFI data to estimate the $\mathrm{CH}$ abundance in the NGC 6334I hot core and constrain the physical properties of the $\mathrm{CH}$ absorbing foreground clouds.

\section{Observations and data reduction}

We use data from a spectral scan from the "Chemical HErschel Survey of Star forming regions" key program (CHESS; Ceccarelli et al. 2010), observed by HIFI on board the Herschel Space Observatory. The band 1a scan was performed on February 28, 2010 using the dual beam switch (DBS) mode with continuum stability optimization. The half-power beamwidth at the relevant frequencies is about $42^{\prime \prime}$ and was centered at $\alpha_{J 2000}=17^{\mathrm{h}} 20^{\mathrm{m}} 53^{\mathrm{s}} .32, \delta_{J 2000}=-35^{\circ} 46^{\prime} 58.5^{\prime \prime}$, which is between the SMA1 and SMA2 cores in Fig. 1 of Hunter et al. (2006). The reference positions for the DBS were $\sim 3^{\prime}$ east and west of the target. HIFI spectral scans use the wide band spectrometer (WBS) with a frequency resolution of $1.1 \mathrm{MHz}$, corresponding to $0.6 \mathrm{~km} \mathrm{~s}^{-1}$ at the frequencies of the two groups of $\mathrm{CH}$ lines. The full band 1a (480-560 GHz) scan took $12950 \mathrm{~s}$, including overheads. The resulting noise level is $12 \mathrm{mK}$ in $T_{\mathrm{mb}}$ units, measured in $0.5 \mathrm{MHz}$ channels, in the "single sideband" spectrum (see below), averaged between $\mathrm{H}$ - and V-polarization. The double sideband system temperature varied between 60 and $110 \mathrm{~K}$.

The raw data is pipelined in HIPE 2.6 (Ott 2010). We note that the absolute velocity scale is uncertain because of improper calculation of the spacecraft motion, but this has been constrained to $0.3 \mathrm{~km} \mathrm{~s}^{-1}$ by subsequent reprocessing in a more recent pipeline version. The resulting level 2 product was processed in $\mathrm{CLASS}^{1}$. After mixing the sky signal with a local oscillator (LO) signal, a heterodyne detector such as HIFI yields a double sideband spectrum. In spectral scans, each frequency channel is observed using several LO settings (observations discussed here use a redundancy of 8 ), which makes it possible to mathematically disentangle the signal from the two separate sidebands (Comito \& Schilke 2002). This "deconvolution" step is executed in CLASS, while allowing channel-to-channel variations in receiver gain. Resulting gain variations are $10 \%$ at maximum. Spectra presented in this Letter are always deconvolved single sideband spectra. Intensities are corrected for a main beam efficiency of $70.8 \%$ expected for band 1a of HIFI.

\section{Line profile analysis}

The single sideband HIFI WBS spectra near 536.76 and $532.73 \mathrm{GHz}$ (Fig. 1) show continuum emission with $T_{\mathrm{mb}}=$ $1.19 \mathrm{~K}$ and several spectral lines. The two spectral segments, each about $0.2 \mathrm{GHz}$ wide, cover the $\mathrm{CH}^{2} \Pi_{3 / 2} J=3 / 2-1 / 2$ parity ${ }^{+}$to ${ }^{-}$transitions around $532.73 \mathrm{GHz}$ and the parity ${ }^{-}$to ${ }^{+}$transitions around 536.76 GHz (Davidson et al. 2001). Most lines are

\footnotetext{
1 Version of April 2010, with some improvements. CLASS is part of the GILDAS software developed at IRAM: http://www.iram.fr/ IRAMFR/GILDAS
}
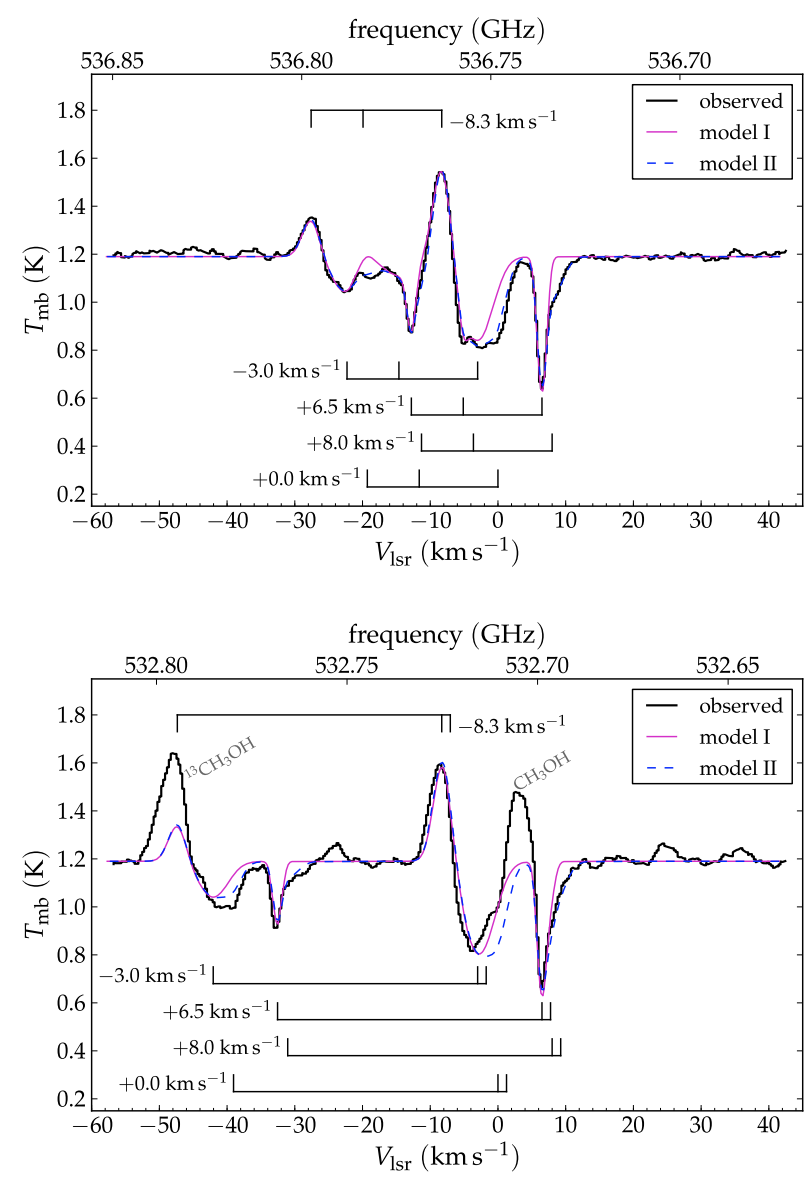

Fig. 1. Observed spectra (black) of the two groups of three hyperfine components of the $\mathrm{CH}^{2} \Pi_{3 / 2} J=3 / 2-1 / 2$ transition, represented in $T_{\mathrm{mb}}$ units along the vertical axis (see Sect. 2). The horizontal axis gives frequency (top) and velocity with respect to the rest frequency for the strongest line in each triplet (bottom). The magenta line represents model I with one emission component and two absorption components and the blue line represents model II with two additional absorption components. Physical parameters of the model components are given in Table 1. "Comb" labels indicate the loci of the $\mathrm{CH}$ hyperfine lines shifted with respect to the rest frequency.

due to $\mathrm{CH}$, but two emission features in the $532.73 \mathrm{GHz}$ spectrum have contributions from the $\mathrm{CH}_{3} \mathrm{OH} J_{K}=15_{2}-15_{1}$ and the ${ }^{13} \mathrm{CH}_{3} \mathrm{OH} 9_{3}-9_{2}$ transitions.

\subsection{Model setup}

From inspection of the observed spectrum in Fig. 1, we note that the two sets of three emission peaks are spaced by exactly the hyperfine splitting. Since we see more than three absorption features near each emission triplet, we conclude that more than one absorption component is necessary to explain the observed spectra. We therefore generate a model $\mathrm{CH}$ spectrum starting from the minimum of one emitting and two absorbing components ("model I", see Table 1) using the CASSIS software ${ }^{2}$ and the CDMS spectroscopic database (Müller et al. 2005). Given an excitation temperature and a column density for a set of emission and absorption lines, the "LTE" module in CASSIS calculates a frequency-dependent optical depth, which is used to construct a beam-averaged, frequency-dependent line brightness.

We adopt a source size of $10^{\prime \prime}$ for the emission component, taken to encompass the four SMA cores (Hunter et al. 2006). With a beam size of $42^{\prime \prime}$ for HIFI band 1a, this results in a beamfilling factor of $6 \%$. Since we lack collisional rate coefficients

\footnotetext{
${ }^{2}$ CASSIS has been developed by CESR-UPS/CNRS (http:// cassis.cesr.fr).
} 
Table 1. Model component parameters.

\begin{tabular}{lccccc}
\hline \hline Component & $T_{\mathrm{ex}}$ & $\begin{array}{c}V_{\mathrm{lsr}} \\
(\mathrm{K})\end{array}$ & $\begin{array}{c}F W H M \\
\left.\mathrm{~km} \mathrm{~s}^{-1}\right)\end{array}$ & \multicolumn{2}{c}{$N_{\mathrm{CH}}\left(10^{13} \mathrm{~cm}^{-2}\right)$} \\
model I & model II \\
\hline Emission & 100 & -8.3 & 3.0 & 20 & 20 \\
Absorption A & 5.5 & -3.0 & 5.0 & 6.8 & 6.6 \\
Absorption B & 5.5 & +6.5 & 1.5 & 3.8 & 3.0 \\
Absorption C & 5.5 & +8.0 & 3.5 & $\ldots$ & 2.1 \\
Absorption D & 5.5 & +0.0 & 3.0 & $\ldots$ & 2.1 \\
\hline
\end{tabular}

for $\mathrm{CH}$, we take the dust temperature of $100 \mathrm{~K}$ from Sandell (2000) and assume this to be equal to the excitation temperature $\left(T_{\text {ex }}\right)$ of the emitting $\mathrm{CH}$ gas in NGC 6334I. We note that, while we assume a single value for $T_{\mathrm{ex}}$, the nominal dust temperatures determined from interferometric observations by Hunter et al. (2006) vary between 33 and $100 \mathrm{~K}$ from core to core, with limits of 20 and $300 \mathrm{~K}$. The continuum level for the model is fixed at the observed level of $1.19 \mathrm{~K}$. For the absorbing foreground components, $T_{\text {ex }}=5.5 \mathrm{~K}$ is based on $\mathrm{H}_{2} \mathrm{O}$ absorption signatures discussed in Emprechtinger et al. (2010).

\subsection{Column density estimates}

Since all features in the $536.76 \mathrm{GHz}$ spectrum (Fig. 1, top panel) can be ascribed to $\mathrm{CH}$, we use this as a starting point to constrain the remaining model parameters for the three components: line of sight velocity $\left(V_{\mathrm{lsr}}\right)$, column density $\left(N_{\mathrm{CH}}\right)$, and full-width at half-maximum of the line $(F W H M)$. The bestfit set of parameters presented in Table 1 (model I) are: emission at $V_{\mathrm{lsr}}=-8.3 \mathrm{~km} \mathrm{~s}^{-1}$ with $F W H M=3.0 \mathrm{~km} \mathrm{~s}^{-1}$ and $N_{\mathrm{CH}}=2.0 \times 10^{14} \mathrm{~cm}^{-2}$ and two absorbing components at -3.0 and $+6.5 \mathrm{~km} \mathrm{~s}^{-1}$, with distinctly different line widths of 5.0 and $1.5 \mathrm{~km} \mathrm{~s}^{-1}$, respectively. After fine-tuning the parameters of the three components, some discrepancies between model I and the observed spectra remain. Extra absorption appears to be required near 536.782, 536.748 and $536.732 \mathrm{GHz}$ (see Fig. 1, top panel). The frequency spacing between these discrepant regions is such that they cannot be explained by a single additional component.

The discrepancies can be resolved by adding two absorbing clouds, both with $T_{\mathrm{ex}}=5.5 \mathrm{~K}$ and $N_{\mathrm{CH}}=2 \times 10^{13}$ : component C with $V_{\mathrm{lsr}}=+8.0 \mathrm{~km} \mathrm{~s}^{-1}$ and $F W H M=3.5 \mathrm{~km} \mathrm{~s}^{-1}$, and component D with $V_{\mathrm{lsr}}=0.0 \mathrm{~km} \mathrm{~s}^{-1}$ and $F W H M=3.0 \mathrm{~km} \mathrm{~s}^{-1}$ (Fig. 1). Upon introducing components $\mathrm{C}$ and $\mathrm{D}$, the column densities of the absorbing components $\mathrm{A}$ and $\mathrm{B}$ need to be slightly decreased to $6.6 \times 10^{13}$ and $3.0 \times 10^{13} \mathrm{~cm}^{-2}$, respectively ("model II", Table 1).

Without specific tailoring of the model to match the $532.73 \mathrm{GHz}$ spectrum, it explains this second set of $\mathrm{CH}$ signatures remarkably well, with the exception of the contamination by $\mathrm{CH}_{3} \mathrm{OH}$ and ${ }^{13} \mathrm{CH}_{3} \mathrm{OH}$ (see Fig. 1, bottom panel). Although the addition of components $\mathrm{C}$ and $\mathrm{D}$ moves the model closer to the spectrum of the $536.76 \mathrm{GHz}$ triplet, the correspondence to the $532.73 \mathrm{GHz}$ spectrum still has some notable discrepancies: the absorption feature near $532.78 \mathrm{GHz}$ is not deep enough, and the absorption signature near $532.72 \mathrm{GHz}$ appears to be shifted by $\sim 2 \mathrm{~km} \mathrm{~s}^{-1}$. The latter mismatch could be alleviated by adding a methanol emission line. Additional contamination by other species is not ruled out at the level of $\sim 0.05 \mathrm{~K}$ line intensity, as illustrated by emission features around both 532.670 and $532.754 \mathrm{GHz}$, and possibly the blue wings of the methanol lines.

\subsection{Uncertainties}

Variations in column density for an individual model component have a roughly linear effect on the equivalent width of the corresponding spectral feature, i.e., doubling $N_{\mathrm{CH}}$ (dividing by two) leads to an emission or absorption line that is twice as strong (weak). Moreover, the column densities that we derive for each component depend directly on the assumed values of $T_{\mathrm{ex}}$. We briefly explore the effect of changing $T_{\mathrm{ex}}$ on the line intensities, which we compensate by adjusting the column density. For the emitting gas, raising $T_{\text {ex }}$ from 100 to $150 \mathrm{~K}$ requires a compensating column density increase of $\sim 35 \%$, while lowering $T_{\text {ex }}$ from 100 to 50 (to 30 ) $\mathrm{K}$ requires a column density decrease of only $20 \%(15 \%)$. Because the $\mathrm{CH}$ transition under consideration becomes optically thick at low $T_{\mathrm{ex}}$, the dependence of column density on $T_{\text {ex }}$ weakens and eventually reverses as $T_{\text {ex }}$ is lowered to $\sim 50 \mathrm{~K}$ and beyond. To illustrate, if $T_{\mathrm{ex}}$ is varied from 150 to 100 to 50 to $30 \mathrm{~K}$, the opacity for the main emission line increases from 0.05 to 0.07 to 0.19 to 0.39 , respectively. The line opacity therefore reacts more strongly than linearly to a change in $T_{\mathrm{ex}}$, resulting in counterintuitive behavior of line strength as a function of $T_{\mathrm{ex}}$. For the cold absorbing gas, an increase in $T_{\mathrm{ex}}$ from 5.5 to $20 \mathrm{~K}$ roughly doubles the column density, and changing $T_{\text {ex }}$ from 5.5 to $2.7 \mathrm{~K}$ results in a column density decrease of $<10 \%$. This illustrates a much weaker dependence of column density on $T_{\mathrm{ex}}$ than for the emitting gas. Optical depths reach maxima of $0.34,0.51,0.15$, and 0.18 at the respective line centers for absorbing components A, B, C, and D.

The uncertainty in our derived column density for the emission component is dominated by the uncertainty in the $T_{\mathrm{ex}}$ estimates $(\lesssim 2$, see Sect. 3.1). Following the above discussion, quoted column density values can be trusted taking into account an error margin of $\sim 30 \%$ for the warm emitting gas. On the other hand, the uncertainty in column densities for the cold absorbing gas components is dominated by measurement errors in the continuum and absorption line depth, each estimated at $6 \%$, resulting in an uncertainty of $8 \%$ for the column densities.

\section{Discussion}

\section{1. $\mathrm{CH}$ in the hot core}

The velocity of the emission component at $-8.3 \mathrm{~km} \mathrm{~s}^{-1}$ is consistent with the $\mathrm{H}_{2} \mathrm{O}$ emission detected by Emprechtinger et al. (2010) at $-8.1 \mathrm{~km} \mathrm{~s}^{-1}$ and close to the velocity of $\approx-7 \mathrm{~km} \mathrm{~s}^{-1}$ of molecular lines measured from the ground (Nummelin et al. 1998). The width of the $\mathrm{CH}$ emission features of $3.0 \mathrm{~km} \mathrm{~s}^{-1}$ is smaller than the $4-5 \mathrm{~km} \mathrm{~s}^{-1}$ for $\mathrm{NH}_{3}$ (ATCA at $24 \mathrm{GHz}$; Beuther et al. 2005), $6.5 \mathrm{~km} \mathrm{~s}^{-1}$ for $\mathrm{SiO}$, and $6 \mathrm{~km} \mathrm{~s}^{-1}$ for $\mathrm{HC}_{3} \mathrm{~N}$ (IRAM $30 \mathrm{~m}$ at 87, 130 and $155 \mathrm{GHz}$; Bachiller \& Cernicharo 1990). This difference suggests that the $\mathrm{CH}$ emission originates in different, less turbulent and/or less evolved, cores than the heavier N- and O-bearing molecules. Lis et al. (2010) find a similarly narrow linewidth of $3.3 \mathrm{~km} \mathrm{~s}^{-1}$ from HIFI observations of $\mathrm{H}^{37} \mathrm{Cl} \mathrm{1-0}$ emission from the NGC 6334I hot core. Sandell (2000) derives a source size of $(10 \times 8)^{\prime \prime}$ and a dust temperature of $100 \mathrm{~K}$ from sub-millimeter dust continuum measurements. Combining this with a $350 \mu \mathrm{m}$ flux from SHARC-II, dust grain opacities from Ossenkopf \& Henning (1994) and the distance of $1.7 \mathrm{kpc}$, we arrive at $N_{\mathrm{H}_{2}} \approx 3 \times 10^{24} \mathrm{~cm}^{-2}$. From this $N_{\mathrm{H}_{2}}$ and our $N_{\mathrm{CH}}=2.0 \times 10^{14} \mathrm{~cm}^{-2}$, we find an abundance for $\mathrm{CH}$ of $7 \times 10^{-11}$ relative to $\mathrm{H}_{2}$ in the hot core. This is a factor 7-30 lower than the $\mathrm{CH}$ abundance for the dense envelope of Sgr B2, and a factor $~ 300$ lower than observed $\mathrm{CH}$ abundances in diffuse clouds (Polehampton et al. 2005, Table 4, and references therein). This decrease in $\mathrm{CH}$ abundance is similar to that predicted by chemical models (Sect. 1) for $\mathrm{H}_{2}$ densities of $\gtrsim 10^{4} \mathrm{~cm}^{-3}$.

\section{2. $\mathrm{CH}$ in absorbing clouds}

The range of turbulent linewidths for our four absorbing clouds (Table 1) and $N_{\mathrm{CH}}$ values of several $10^{13} \mathrm{~cm}^{-2}$ agree with models of "turbulence dissipation regions" (Godard et al. 2009) 
and with optical observations of diffuse clouds (Liszt \& Lucas 2002). Using the range of $N_{\mathrm{CH}}$ values for absorption clouds A-D (Table 1) and an $N_{\mathrm{CH}} / N_{\mathrm{H}_{2}}$ ratio of $3.5 \times 10^{-8}$ (Sheffer et al. 2008), we find $N_{\mathrm{H}_{2}}=(0.6-2) \times 10^{21} \mathrm{~cm}^{-2}$. Dividing $N_{\mathrm{H}_{2}}$ of $10^{21} \mathrm{~cm}^{-2}$ by a number density of $10^{2}-10^{3} \mathrm{~cm}^{-3}$ applicable to diffuse gas, we derive a rough linear size estimate of $0.3-3 \mathrm{pc}$ for the absorbing clouds. We also convert $N_{\mathrm{H}_{2}}=(0.6-$ 2) $\times 10^{21} \mathrm{~cm}^{-2}$ into a visual extinction, $A_{V} \sim 0.3-1$, based on $N_{\mathrm{H}} / A_{V}=1.87 \times 10^{21} \mathrm{~cm}^{-2} \mathrm{mag}^{-1}$ (Bohlin et al. 1978). Hence, we conclude that the $\mathrm{CH}$ absorption components are diffuse interstellar clouds. We note that, although we discuss several absorbing components as separate entities in velocity space, a diffuse interstellar cloud of several parsecs in size can enjoy a velocity gradient of a few $\mathrm{km} \mathrm{s}^{-1}$ (cf. FWHM in Table 1) and therefore various components may originate in a single molecular cloud.

Other studies also find absorbing gas along the line of sight toward NGC 6334I. We summarize those that potentially correspond to our $\mathrm{CH}$ clouds. Brooks \& Whiteoak (2001) note two clouds absorbing in $\mathrm{OH}$ (near $1.6 \mathrm{GHz}$ ) toward several NGC 6334 cores: one cloud with velocities ranging between -15 and $+2 \mathrm{~km} \mathrm{~s}^{-1}$, which they assume to be associated with the NGC 6334 complex, and another at $+6 \mathrm{~km} \mathrm{~s}^{-1}$ which they ascribe to a more extended foreground cloud. In addition, the HIFI study of $\mathrm{H}_{2} \mathrm{O}$ lines toward NGC 6334I by Emprechtinger et al. (2010) reports absorbing foreground clouds at $-6.3,-0.1$ and $+6.1 \mathrm{~km} \mathrm{~s}^{-1}$.

In terms of $V_{\mathrm{lsr}}$, our absorption components $\mathrm{A}$ and $\mathrm{D}$ (Table 1) fit in the widespread $[-15,+2] \mathrm{km} \mathrm{s}^{-1} \mathrm{OH}$ absorption clouds, which places them physically close to the NGC 6334 molecular cloud. In addition, cloud D coincides with one of the three $\mathrm{H}_{2} \mathrm{O}$ absorbing clouds. Hence, although $N_{\mathrm{CH}}$ for cloud A is three times higher than for cloud $\mathrm{D}$, we conclude that cloud $\mathrm{D}$ must be of somewhat higher number density to allow for $\mathrm{H}_{2} \mathrm{O}$ formation. Moreover, Ossenkopf et al. (2010) find $\mathrm{H}_{2} \mathrm{O}^{+}$absorption toward NGC 6334I. There is notable uncertainty regarding the rest frequencies of the $\mathrm{H}_{2} \mathrm{O}^{+}$ground state rotational transitions. We assume a value of $1115.209 \mathrm{GHz}$ for $F=5 / 2-3 / 2$, which is close to predictions by Mürtz et al. (1998), to derive $V_{\text {lsr }} \approx-1 \mathrm{~km} \mathrm{~s}^{-1}$ for the $\mathrm{H}_{2} \mathrm{O}^{+}$absorption. Both the above $\mathrm{H}_{2} \mathrm{O}^{+}$ signature and the detection of $\mathrm{H}_{2} \mathrm{Cl}^{+}$absorption at $-1.7 \mathrm{~km} \mathrm{~s}^{-1}$ with a width of $12 \mathrm{~km} \mathrm{~s}^{-1}$ (Lis et al. 2010) are consistent with a blend of our $\mathrm{CH}$ components $\mathrm{A}$ and $\mathrm{D}$ in velocity space.

Our component B at $+6.5 \mathrm{~km} \mathrm{~s}^{-1}$ is consistent with both the $+6.1 \mathrm{~km} \mathrm{~s}^{-1} \mathrm{H}_{2} \mathrm{O}$ absorption (Emprechtinger et al. 2010) and the $+6 \mathrm{~km} \mathrm{~s}^{-1} \mathrm{OH}$ component to within the $0.7 \mathrm{~km} \mathrm{~s}^{-1}$ velocity resolution of the ATCA observations (Brooks \& Whiteoak 2001). We believe that this cloud has a relatively high particle density, but is unrelated to the NGC 6334 complex. Cloud C is seen in $\mathrm{CH}$ but has no counterparts in $\mathrm{H}_{2} \mathrm{O}$ or $\mathrm{OH}$. The column density of this cloud is not particularly low with respect to clouds A, B, and D (cf. Table 1), but it might simply be of lower number density. In conclusion, from the correspondence with counterparts in other molecules, we find that $\mathrm{CH}$ clouds $\mathrm{B}$ and $\mathrm{C}$ are unrelated foreground clouds, while clouds $\mathrm{A}$ and $\mathrm{D}$ are located in the direct surroundings of NGC 6334I.

\subsection{Dense clouds and outflows}

In addition to the absorbing clouds discussed in Sect. 4.2, the literature provides a collection of absorption signatures without $\mathrm{CH}$ counterparts. First, we find no evidence of absorbing $\mathrm{CH}$ gas that matches the $-6.3 \mathrm{~km} \mathrm{~s}^{-1}$ component seen in $\mathrm{H}_{2} \mathrm{O}$ (Emprechtinger et al. 2010). The low $\mathrm{CH}$ abundance in this cloud - close to the NGC 6334I hot core in velocity space - may be due to its physical proximity to the luminous NGC 6334 region, Page 4 of 5 where higher gas densities facilitate active chemistry, leading to depletion of $\mathrm{CH}$. In general, overall column density and visual extinction are traced more accurately by $\mathrm{CH}$ than by $\mathrm{H}_{2} \mathrm{O}$ and $\mathrm{CO}$, which are very sensitive to inhomogeneity (e.g., Spaans 1996).

Second, Beuther et al. (2005) detect $\mathrm{CH}_{3} \mathrm{OH}$ and $\mathrm{NH}_{3}$ absorption at $25 \mathrm{GHz}$ toward NGC 6334-I-SMA3 (the compact H II region) at $-9 \mathrm{~km} \mathrm{~s}^{-1}$, about $2 \mathrm{~km} \mathrm{~s}^{-1}$ blueward of the emission component. Moreover, outflows with velocities up to $50 \mathrm{~km} \mathrm{~s}^{-1}$ are observed in $\mathrm{H}_{2} \mathrm{O} 1_{11}-0_{00}, 2_{11}-2_{02}, 1_{10}-1_{01}$ and $3_{12}-3_{03}$ emission (Emprechtinger et al. 2010) and up to $70 \mathrm{~km} \mathrm{~s}^{-1}$ in $\mathrm{CO}$ $J=2-1,3-2,4-3$ and 7-6 (Bachiller \& Cernicharo 1990; Leurini et al. 2006). In our HIFI observations of the $\mathrm{CH}$ transition, we detect neither blueshifted gas nor outflows to match the $\mathrm{H}_{2} \mathrm{O}, \mathrm{CH}_{3} \mathrm{OH}, \mathrm{NH}_{3}, \mathrm{CO}$ and $\mathrm{H}_{2} \mathrm{O}^{+}$, in neither emission nor absorption. The absence of $\mathrm{CH}$ in both blueshifted emission and outflows indicates that $\mathrm{CH}$ is destroyed in shocked environments. We conclude that $\mathrm{CH}$ traces molecular material that has not yet been swept up by outflow activity related to star formation.

\section{Conclusions}

We use $\mathrm{CH}$ as a tracer of cold, quiescent, molecular gas in both the star-forming core of NGC 6334I and diffuse clouds along the line of sight. We derive a very low $\mathrm{CH}$ abundance of $7 \times 10^{-11}$ in the hot core, consistent with $\mathrm{CH}$ depletion in chemically active dense cores. Evidence is found of four absorbing components composed of diffuse gas. From comparison with other molecular absorption signatures in the literature, we conclude that clouds A and D are related to the NGC 6334 complex, and that clouds B and $\mathrm{C}$ are unrelated foreground clouds (Table 1). Differences between the four clouds in terms of counterparts in other molecular tracers are ascribed to local variations in density and dynamics. Dynamical stirring of the gas is hypothesized to be the cause of the absence of $\mathrm{CH}$ in the outflow component, which is detected in other molecular tracers.

\section{References}

Adams, W. S. 1941, ApJ, 93, 11

Bachiller, R., \& Cernicharo, J. 1990, A\&A, 239, 276

Beuther, H., Thorwirth, S., Zhang, Q., et al. 2005, ApJ, 627, 834 Bohlin, R. C., Savage, B. D., \& Drake, J. F. 1978, ApJ, 224, 132 Brooks, K. J., \& Whiteoak, J. B. 2001, MNRAS, 320, 465

Ceccarelli, C., Bacmann, A., Boogert, A., et al. 2010, A\&A, 521, L22 Chastain, R. J., Cotten, D., \& Magnani, L. 2010, AJ, 139, 267

Comito, C., \& Schilke, P. 2002, A\&A, 395, 357

Davidson, S. A., Evenson, K. M., \& Brown, J. M. 2001, ApJ, 546, 330 de Graauw, Th., Helmich, F. P., Philips, T. G., et al. 2010, A\&A, 518, L6 Emprechtinger, M., Lis, D. C., Bell, T., et al. 2010, A\&A, 521, L28 Godard, B., Falgarone, E., \& Pineau Des Forêts, G. 2009, A\&A, 495, 847 Hunter, T. R., Brogan, C. L., Megeath, S. T., et al. 2006, ApJ, 649, 888 Leurini, S., Schilke, P., Parise, B., et al. 2006, A\&A, 454, L83 Lis, D. C., Pearson, J. C., Neufeld, D. A., et al. 2010, A\&A, 521, L9 Liszt, H., \& Lucas, R. 2002, A\&A, 391, 693

Müller, H. S. P., Schlöder, F., et al. 2005, J. Molec. Struct., 742, 215 Mürtz, P., Zink, L. R., et al. 1998, J. Chem. Phys., 109, 9744

Nummelin, A., Dickens, J. E., Bergman, P., et al. 1998, A\&A, 337, 275 Ossenkopf, V., \& Henning, T. 1994, A\&A, 291, 943

Ossenkopf, V., Müller, H. S. P., Lis, D. C., et al. 2010, A\&A, 518, L111 Ott, S. 2010, in ADASS XIX, ed. Y. Mizumoto, \& M. Ohishi, in press Pilbratt, G. L., Riedinger, J. R., Passvogel, T., et al. 2010, A\&A, 518, L1 Polehampton, E. T., Menten, K. M., Brünken, S., et al. 2005, A\&A, 431, 203 Rydbeck, O. E. H., Elldér, J., \& Irvine, W. M. 1973, Nature, 246, 466 Sandell, G. 2000, A\&A, 358, 242

Schilke, P., Comito, C., Thorwirth, S., et al. 2006, A\&A, 454, L41 Sheffer, Y., Rogers, M., Federman, S. R., et al. 2008, ApJ, 687, 1075 Spaans, M. 1996, A\&A, 307, 271

Stacey, G. J., Lugten, J. B., \& Genzel, R. 1987, ApJ, 313, 859

Turner, B. E., \& Zuckerman, B. 1974, ApJ, 187, L59+

Van Dishoeck, E. F., \& Black, J. H. 1986, ApJS, 62, 109 
Acknowledgements. HIFI has been designed and built by a consortium of institutes and university departments from across Europe, Canada and the United States under the leadership of SRON Netherlands Institute for Space Research, Groningen, The Netherlands and with major contributions from Germany, France and the US. Consortium members are: Canada: CSA, U. Waterloo; France: CESR, LAB, LERMA, IRAM; Germany: KOSMA, MPIfR, MPS; Ireland, NUI Maynooth; Italy: ASI, IFSI-INAF, Osservatorio Astrofisico di Arcetri-INAF; Netherlands: SRON, TUD; Poland: CAMK, CBK; Spain: Observatorio Astronómico Nacional (IGN), Centro de Astrobiología (CSIC-INTA). Sweden: Chalmers University of Technology - MC2, RSS \& GARD; Onsala Space Observatory; Swedish National Space Board, Stockholm University - Stockholm Observatory; Switzerland: ETH Zürich, FHNW; USA: Caltech, JPL, NHSC. We thank Marco Spaans for useful discussions, and the referee Volker Ossenkopf for constructive suggestions.

1 Kapteyn Astronomical Institute, University of Groningen, PO Box 800, 9700AV, Groningen, The Netherlands e-mail: wiel@astro.rug.nl

2 SRON Netherlands Institute for Space Research, Groningen, NL

3 California Institute of Technology, Pasadena, USA

4 Department of Astronomy, University of Michigan, Ann Arbor, USA

5 Max-Planck-Institut für Radioastronomie, Bonn, Germany

${ }^{6}$ Physikalisches Institut, Universität zu Köln, Köln, Germany

7 Centre d'Étude Spatiale des Rayonnements, Université Paul Sabatier, Toulouse, France

8 CNRS/INSU, UMR 5187, Toulouse, France

9 Laboratoire d'Astrophysique de Grenoble, UMR 5571-CNRS, Université Joseph Fourier, Grenoble, France
10 Université de Bordeaux, Laboratoire d'Astrophysique de Bordeaux, Floirac, France

11 CNRS/INSU, UMR 5804, Floirac Cedex, France

12 Jet Propulsion Laboratory, Caltech, Pasadena, CA 91109, USA

13 Ohio State University, Columbus, OH, USA

14 INAF - Istituto di Fisica dello Spazio Interplanetario, Roma, Italy

15 Infared Processing and Analysis Center, Caltech, Pasadena, USA

16 Johns Hopkins University, Baltimore MD, USA

17 Laboratoire d'Études du Rayonnement et de la Matière en Astrophysique, UMR 8112 CNRS/INSU, OP, ENS, UPMC, UCP, Paris, France

18 School of Physics and Astronomy, University of Leeds, Leeds UK

19 Centro de Astrobiologìa, CSIC-INTA, Madrid, Spain

20 INAF Osservatorio Astrofisico di Arcetri, Florence, Italy

21 Astronomical Institute "Anton Pannekoek", University of Amsterdam, Amsterdam, The Netherlands

22 Department of Astrophysics/IMAPP, Radboud University Nijmegen, Nijmegen, The Netherlands

23 IGN Observatorio Astronómico Nacional, Alcalá de Henares, Spain

24 Max-Planck-Institut für Astronomie, Heidelberg, Germany

25 Harvard-Smithsonian Center for Astrophysics, Cambridge MA, USA

${ }^{26}$ INAF - Osservatorio Astronomico di Roma, Monte Porzio Catone, Italy

27 Institut de RadioAstronomie Millimétrique, Grenoble, France

28 Leiden Observatory, Leiden University, Leiden, The Netherlands

29 Department of Physics and Astronomy, University College London, London, UK

${ }^{30}$ Dept. of Physics and Astronomy, University of Waterloo, Canada

31 European Space Astronomy Centre, ESA, Madrid, Spain 